\title{
Special issue on recent progress with hPSC-derived cardiovascular cells for organoids, engineered myocardium, drug discovery, disease models, and therapy
}

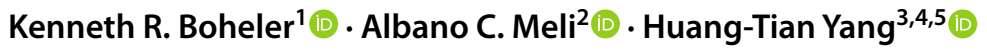 \\ Received: 5 June 2021 / Revised: 5 June 2021 / Accepted: 9 June 2021 /Published online: 16 June 2021 \\ (c) The Author(s), under exclusive licence to Springer-Verlag GmbH Germany, part of Springer Nature 2021
}

Over 35 years ago, Doetschman et al. were the first to describe the in vitro differentiation of cardiomyocytes (CMs) from mouse embryonic stem cells (mESCs) [7]. Then, just as today, the leading causes of adult morbidity and mortality in Western Societies were cardiovascular diseases [2]. Doetschman's original findings and those subsequently from other investigators led to the hope that in vitro derived CMs could facilitate the improved study of mammalian cardiac development, the creation of new and informative cardiac disease models, and advance testing of drug efficacy and toxicology [37]. Initially, the in vitro differentiated CMs from $\mathrm{mESCs}$ were described as being small and immature, retained some proliferative capacity, and displayed a developmental continuum in terms of ion channel expression and function. With time, these cells facilitated a reductionist biology approach; i.e., the mESC lines were amenable

This article is part of the special issue on "hPSC-derived Cardiovascular Cells for Organoids, Engineered Myocardium, Drug Discovery, Disease Models and Therapy" in Pflügers Archiv-European Journal of Physiology

Kenneth R. Boheler

kbohele1@jhmi.edu

Albano C. Meli

albano.meli@inserm.fr

$\triangle$ Huang-Tian Yang

htyang@sibs.ac.cn

1 Department of Biomedical Engineering, Whiting School of Engineering, The Johns Hopkins University, Baltimore, MD 21205, USA

2 PhyMedExp, University of Montpellier, INSERM, CNRS, Montpellier, France

3 CAS Key Laboratory of Tissue Microenvironment \& Tumor, Laboratory of Molecular Cardiology, Shanghai Institute of Nutrition and Health, University of Chinese Academy of Sciences (CAS), CAS, Shanghai 200031, People's Republic of China to genetic manipulation, which facilitated the analysis of specific gene defects on developmental processes in vitro and in vivo $[4,36]$. While these mouse model systems were instrumental in determining some genetic causes of human cardiac disease, profound physiological and developmental differences between mouse and human (e.g., beating rates, action potentials, ion channel expression, and current profiles) confounded data interpretation.

Ultimately, it was the generation of human ESC (hESC) lines and the emergence of human induced pluripotent stem cells (hiPSCs) that led to an explosion in research activity [31, 32]. Human pluripotent stem cell (hPSC)-derived cardiovascular cells held promise not only for developmental studies and model development of human cardiac disorders that are often complex, polygenic conditions strongly influenced by environmental and genetic factors, but also for cardiac repair and regenerative medicine $[6,8,16,23,36]$. From a basic science perspective, these hPSC derivatives had numerous advantages over other mammalian systems for the cellular study of development, physiology, disease,

4 Translational Medical Center for Stem Cell Therapy \& Institute for Heart Failure and Regenerative Medicine, Shanghai East Hospital, Tongji University School of Medicine and Shanghai Institute of Stem Cell Research and Clinical Translation, Shanghai 200123, People's Republic of China

5 Institute for Stem Cell and Regeneration, CAS, Beijing 100101, People's Republic of China 
and pharmaceuticals. Similar to mESCs, human PSCs are amenable to gene targeting and gene editing using advanced technologies (e.g., CRISPR/Cas 9) [10, 34]. Advances with hPSCs and the generation of isogenic controls have already improved our understanding of genetic factors that contribute to development, rhythm disorders, cardiomyopathies, and valvular and vascular disorders and to metabolic risk factors contributing to cardiomyopathies and ischemic heart disease $[24,26]$. During the course of these studies, however, a number of limitations have been identified that have tempered the great expectations for human PSC-derived cardiomyocytes (hPSC-CMs), particularly for regenerative medicine [17, 24]. As noted with mouse, human PSC-CMs are generally immature and retain traits most typical of embryonic or fetal cardiomyocytes [28]. These cells in two-dimensional (2D) cultures also undergo a developmental block in vitro that limits their ability to form more mature, adult-like CMs suitable for drug screening and for model development of adult syndromes [12, 21, 22, 33]. When transplanted into suitable mammalian model systems, only very early in vitro differentiated cells retain the ability of forming cardiomyocytes with "adult-like" morphologies with well-defined contractile properties [11, 12, 29]. Problematically, immature in vitro differentiated hPSC-CMs transplanted into adult hearts contribute to arrhythmias, which may limit their regenerative potential [19]. Genome-wide profiling has shown that $5-46 \%$ of hiPSC phenotype variability, including differentiation capacity and cellular morphology, results from differences among individuals [14]. We now recognize that an understanding of the mechanisms underlying cardiovascular cell biology requires defined cell types with knowledge of the molecular species present, how they interact, and how they respond to stimuli both at a cellular and a complex organismal level $[3,12]$. Furthermore, studies of molecular and cellular cardiology are challenged by the diversity, complexity, heterogeneity, and dynamic range of cells and molecules present within the cardiovascular system that result in experimental bias according to the constraints of the approaches used. Recent advances in technologies that improve our ability to identify, quantify, localize, characterize, and manipulate the cells, their genomes, their metabolism, and their carbohydrates are paramount in determining how they behave in health and disease.

New cell applications and the emergence of innovative technologies used in the study of hPSC-CMs for basic biology, bioengineering, and regenerative medicine are rapidly emerging. In this special issue entitled "Recent Progress with hPSC-derived Cardiovascular Cells for Organoids, Engineered Myocardium, Drug Discovery, Disease Models and Therapy," a series of reviews, research perspectives, and primary research articles summarize the current state of hPSC-CM research and describe emerging technologies that are likely to overcome existing limitations to the use of
hPSC-CMs for research and clinical applications. In choosing these topics, it was our aim to introduce the readers to both the current state of research as well as to cutting edge approaches that may represent the driving force for new discoveries and physiological applications of human stem cells to the cardiovascular system.

Issues of cell heterogeneity are addressed in a review by Boheler and Poon [5]. These authors provide an exhaustive overview of some of the current limitations associated with hPSC-CM heterogeneity arising from in vitro differentiation protocols. This includes issues of contaminating non-cardiomyocyte cells and issues of maturation, genetic variability due to diversity among hiPSC lines, cell reprogramming, and culture conditions. The authors describe the heterogeneity in cardiac maturation and current efforts to promote in vitro maturation. They describe current approaches to sort hPSC-CM from non-cardiomyocytes using non-genetic strategies, including the use of the mitochondrial potentiometric dye, lactate metabolism in glucose- and pyruvate-free conditions, temperature control for hPSC-CM enrichment, as well as molecular beacons. The authors then describe how immunophenotyping coupled with cell sorting is a validated approach for overcoming many of these limitations of heterogeneity and provide an exhaustive review of mostly positive selection techniques. Highlights include discussions of cell surface proteins like activated leukocyte cell adhesion molecule (ALCAM), signal regulatory protein alpha (SIRPA, CD172a), and vascular cell adhesion protein 1 (VCAM1) for cardiomyocyte purification and CD36 for isolation of cardiomyocytes with a defined maturation state.

In the research article by Luo et al. [20], the authors used the fluorescent protein Venus to determine whether myosin light chain 2 protein (MLC2v) could serve as a useful marker to distinguish between atrial and ventricular $\mathrm{CMs}$ derived from hESCs. This is relevant as MYL2 in mouse is present developmentally in the atrioventricular canal and outflow tract, even though MYL2 is dominantly localized to ventricular myocardium [25]. The authors report a developmental continuum of MYL2 expression of hESC-CMs. At early differentiation stages, both MLC2 $\mathrm{v}$-Venus ${ }^{-}$and MLC2v-Venus ${ }^{+} \mathrm{CMs}$ displayed ventricular-like traits, but the ventricular-like cells from MLC2v-Venus ${ }^{+} \mathrm{hESC}-\mathrm{CMs}$ displayed more developed action potential (AP) properties than those from MLC2v-Venus ${ }^{-}$hESC-CMs. About half of the MLC2 $\mathrm{v}-\mathrm{Venus}^{-} \mathrm{CMs}$ also displayed traits typical of atrial-like cells. At later stages, almost all MLC2v-Venus ${ }^{+}$ cells have ventricular-like characteristics, and relative to the MLC2v-Venus ${ }^{-}$cells, the Venus ${ }^{+}$cells had improved sarcomeric structures, calcium handling properties, and mitochondrial function. The MLC2v-Venus + cells were also more sensitive to hypoxic stress. Their findings suggest that early expression of MYL2 may be informative as an early marker of chamber-specific cardiomyocytes. In a separate 
research article by Giannetti et al. [9], the authors perform a comprehensive study of the hyperpolarization-activated "funny" current $\left(\mathrm{I}_{\mathrm{f}}\right)$ in hiPSC-derived CMs with pacemaker activity. This is a timely publication, as the $\mathrm{I}_{\mathrm{f}}$ channel is expressed in hPSC-CMs well after the initiation of spontaneous contractions. Their extensive electrophysiological analyses coupled with pharmacological interventions firmly establish the pacemaker CMs as a suitable model for studying human $\mathrm{I}_{\mathrm{f}}$-related sinus arrhythmias.

A new and evolving field of research that is poorly defined is glycomics, which is the comprehensive study of the entire complement of sugars present on complex molecules. Because the process of glycosylation depends on cell type and development, the study of glycomics may have profound biological significance on pathways critical for differentiation and maturation of hPSC-CMs. To address this issue, Kelly et al. [13] contributed a perspective article consisting of a review of protein glycosylation ( $\mathrm{N}$ - and O-linked) coupled with new experimental results showing the dynamics of glycosylation in hPSC-CMs with time of in vitro differentiation. The authors provide a comprehensive overview of protein glycosylation, and they discuss how protein glycosylation is affected during CM development and in disease. They discuss strategies required to assess protein glycosylation and review published reports on protein glycosylation during hPSC-CM differentiation. They make a compelling case describing how protein glycosylation is dynamic in hPSC-CMs and how changes in protein glycosylation may critically impact hPSC-CM function and maturation. By combining their findings with RNA-seq analyses, they further demonstrate that proteins and enzymes critical for glycosylation (e.g., beta-galactoside alpha-2,3-sialytransferase 4) are dynamically present in hPSC-CMs with time in vitro differentiation. These findings offer mechanistic insights into how glycan structures vary with time of cultivation and contribute to functional heterogeneity.

Applications of hiPSC-derived CMs for drug screening are provided by Andrysiak et al. [1] and by Liu et al. [18] who emphasized the fact that potential therapeutic compounds must be validated during preclinical and clinical trials. To overcome the expense and differences between animal models and humans, cellular models should facilitate high-throughput screening of chemical compounds. Primary human cardiac cells, however, are difficult to obtain, except when differentiated from hPSC lines. Andrysiak et al. [1] discuss the current state of in vitro differentiation of hPSCs to ventricular-, atrial-, and pacemaker-like CMs and described methods to improve CM maturity and functionality. They advocate for the use of hPSC-CMs in conjunction with 3D cardiac structures, including organoids, and 2D heart on a chip models that can promote cell maturation and thus are advantageous for drug screening. The assessment of drugs using hPSC-CMs requires extensive analyses, including electrophysiological techniques, calcium imaging microscopy, measurement of the cellular contractile properties, and gene expression profile. The authors argue that a comprehensive approach is necessary to truly model cardiac disorders and to delineate possible drug effects using high throughout testing of drug libraries on cardiac 2D and 3D models apt for the study of diverse cardiac diseases. From a different perspective, Liu et al. [18] discuss the lack of suitable humanized preclinical models for drug discovery and development. They review underlying mechanisms implicated in the development of ischemic, inherited, and drug-induced heart disease and then discuss the value of hPSC-CMs to model cardiovascular disorders and for preclinical drug screening. They discuss the relevance of specific hPSCs-based cardiac disease models to understand the complexities of inherited cardiac diseases, including monogenic channelopathies and cardiomyopathies as well mitochondrial cardiac disease. Moreover, they argue that the metabolic state of hPSCs-CMs for cardiac disease modeling is of relevance not only to pathological conditions like metabolic cardiomyopathy induced by type 2 diabetes mellitus, but also for cardiotoxicities induced by chemotherapeutic drugs such as doxorubicin and trastuzumab. They conclude by discussing the challenges associated with hPSC-CMs, and they provide emerging solutions, such as the use of 3D organoid structures and heart-on-a-chip, that may overcome some of these problems.

Svobodova et al. [30] and King et al. [15] review the use of hPSC-CMs in disease models and discuss how improved physiological models that incorporate biomechanical environments with hPSC-models may advance the field. Svobodova et al. [30] utilized Duchenne muscle dystrophy (DMD) as an exemplar for iPSC cardiac disease modeling. DMD is an X-linked disease with either dysfunctional or an absence of dystrophin to cause compromised skeletal and cardiac muscle function. Importantly, the cardiac syndrome associated with DMD is poorly mimicked in mouse models, thus illustrating how and why hiPSC-derived models are so valuable to the understanding of disease processes. In vitro, DMD cardiomyocytes from human display modified mechanical and electro-chemical properties, as well as intracellular calcium handling disturbances; however, the hiPSC-derived DMD CMs suffer from incomplete maturation. The authors go on to discuss how these cells may be apt for future therapeutic applications and for patient-targeted drug treatments. King et al. [15] review the physiological relevance of vascularized stem cell derived myocardium and discuss how the generation of biomechanically compatible environments are necessary for improved human models. This comprehensive review discusses cell mechanics, microvasculature, and the convergence of tissue engineering with stem cell biology. They discuss how biomechanics drive human iPSC-derived cardiomyocyte maturation and how 
Fig. 1 Human pluripotent stem cells-derived cardiomyocytes (hPSC-CMs) for drug discovery, organoids, disease models, and therapy. Human PSCs can be generated from healthy individuals or patients and edited to create mutant cell lines or to correct existing mutations. The identification of protein glycosylation and cell markers in hPSC-CMs, the construction of organoids as well as heart-on-a-chip, and the engineered myocardium further expand potential applications of hPSC-CMs in early cardiac development, drug screening, disease modeling, and cardiac therapy

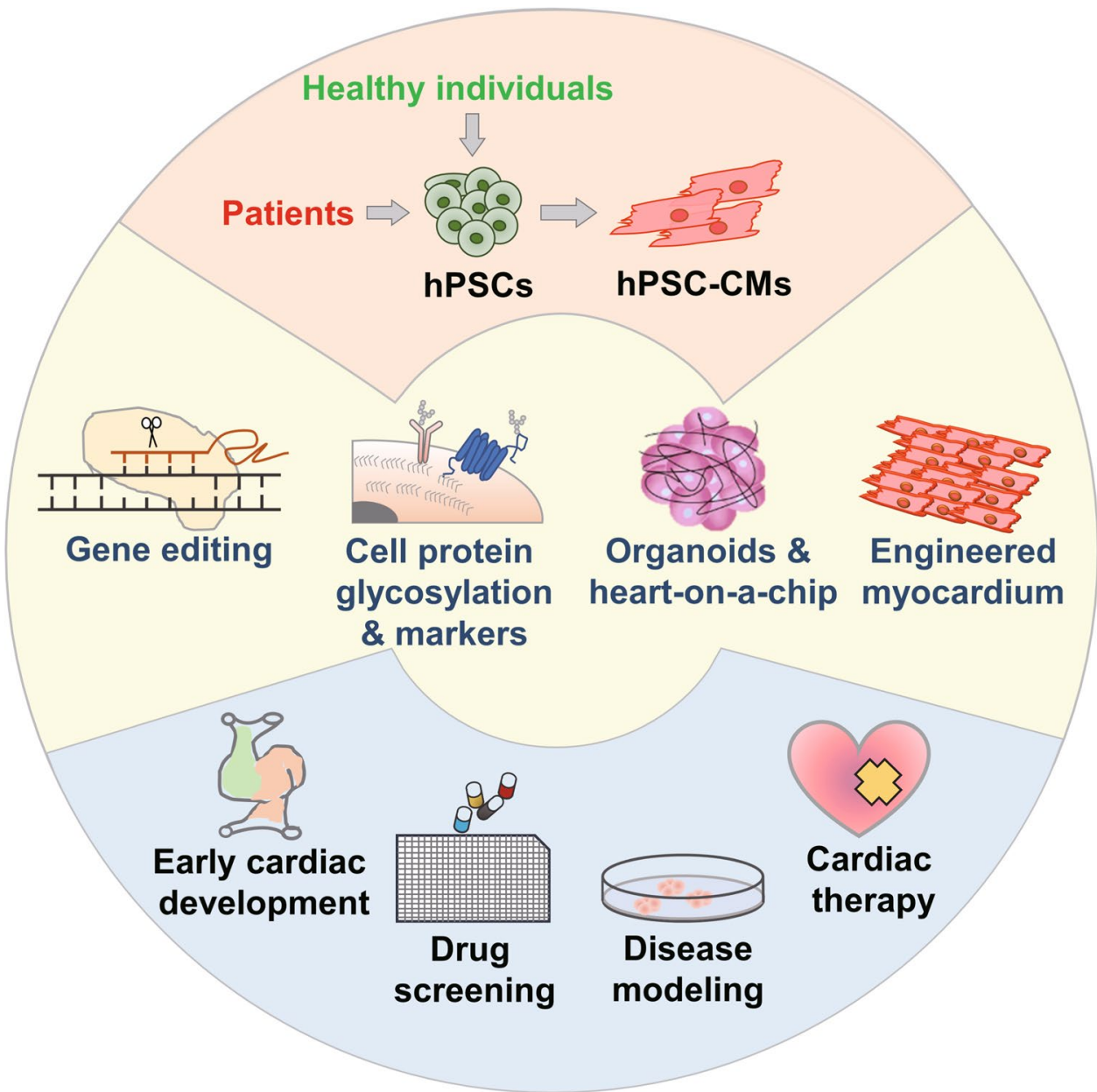

interactions between myocardial and microvascular components are necessary to recapitulate physiological biomechanics in vitro. Additional topics covered in this review include discussions of cell polarization, cell-cell communication, and crosstalk. They also discuss how microfluidic systems and bioprinting can promote better endothelialcardiomyocyte interactions. By using complex bioengineering approaches to create physically relevant biomechanical conditions, they posit that researchers are moving closer to the creation of vascularized human myocardial models with biomechanics that better approximate those of human hearts.

Finally, topics focused on precision medicine are described by Wang et al. [36] and Pan et al. [27]. Precision medicine is best described as "an emerging approach for disease treatment and prevention that takes into account individual variability in genes, environment, and lifestyle" (https://ghr.nlm.nih.gov/primer/precisionmedicine/defin ition). Wang et al. [36] describe the use of patient-specific iPSCs for the study of cardiomyopathies (involving structural and non-structural proteins), cardiac channelopathies (including those that involve potassium and sodium channels), and other cardiovascular diseases (e.g., DMD, supravalvular aortic stenosis, and pulmonary arterial hypertension). They discuss the benefits of employing engineered heart tissues for disease modeling and how these systems can be invaluable for drug screening using patient-specific iPSC lines. Pan et al. [27] expand upon this subject by discussing the value of using hiPSC-CM models for the study of rare cardiovascular diseases (RCDs), which affect millions of people worldwide. Although not always uncommon, many of these RCDs are misdiagnosed or overlooked, which creates obstacles to effective treatment. In the past, studies of these rare diseases have often been limited to the use of patient samples or animal models, but the use of hiPSC-CMs has been critical to recent advances for several channelopathies (such as long QT syndrome, short QT syndrome, Brugada syndrome, and catecholaminergic polymorphic ventricular tachycardia), idiopathic cardiomyopathies, and lysosomal storage diseases. The authors go on to indicate how these diseases can be better understood using precision medicine-based approaches that rely on hiPSC model systems.

In conclusion, we hope that the readers of this special issue will benefit from our selection of topics describing 
the current and evolving state of hPSC-derived cardiovascular cells for organoids, engineered myocardium, drug discovery, and disease models (Fig. 1). Of significance, the original research articles included in this issue are meant to stimulate insights into the characteristics of protein glycosylation and cell markers, as well as in the unique value of hPSC-CMs generated from healthy individuals or patients in drug discovery, disease modeling, and therapy. A better understanding of the heterogeneity of the hPSC-CM pool should also promote the development of novel and relevant approaches for generating and characterizing cardiomyocyte subtypes, as in the analysis of the $\mathrm{I}_{\mathrm{f}}$ channel. Progress in the construction of hPSC-CM with heterotypic endothelial cell base organoids, heart-on-a-chip, and engineered myocardium will ultimately reveal new insights regarding cardiac development, physiology, drug screening, cardiac disease modeling, and therapy, well beyond the purview of 2D cultures or single hPSC-CMs analyses. By sharing the information on emerging technologies (e.g., glycomics) and on developing technologies necessary to promote research excellence in a more time- and cost-effective manner, this collection should assist readers in the development of innovative research strategies to overcome many of the limitations surrounding the current use of hPSC-CMs. Finally, we suggest that these and other advances will contribute to faster translation of research findings from hPSC-CMs into clinical applications.

Acknowledgements We thank Xiao-Ling Luo and Yun Jiang from Shanghai Institute of Nutrition and Health, CAS, for providing technical support to the figure.

Funding Dr. Boheler KR was founded by grants from the National Institutes of Health (NHLBI HL152249-01) and the Huey Foundation; Dr. Yang HT was founded by the National Key R\&D Program of China (2017YFA0103700), the Strategic Priority Research Program of the CAS (No. XDA16010201), and Major Program of Development Fund for Shanghai Zhangjiang National Innovation Demonstration Zone (ZJ2018-ZD-004).

\section{Declarations}

Conflict of interest The authors declare no competing interests.

\section{References}

\section{The volume and page information for the SI article are needed to add after fixed by publisher}

1. Andrysiak K, Stępniewski J, Dulak J (2021) Human-induced pluripotent stem cell-derived cardiomyocytes, 3D cardiac structures, and heart-on-a-chip as tools for drug research. Pflügers Arch - Eur J Physiol. https://doi.org/10.1007/s00424-021-02536-Z

2. Benjamin EJ, Muntner P, Alonso A, Bittencourt MS, Callaway CW, Carson AP, Chamberlain AM, Chang AR, Cheng S, Das SR, Delling FN, Djousse L, Elkind MSV, Ferguson JF, Fornage M, Jordan LC, Khan SS, Kissela BM, Knutson KL, Kwan TW, Lackland DT, Lewis TT, Lichtman JH, Longenecker CT, Loop MS, Lutsey PL, Martin SS, Matsushita K, Moran AE, Mussolino ME, O'Flaherty M, Pandey A, Perak AM, Rosamond WD, Roth GA, Sampson UKA, Satou GM, Schroeder EB, Shah SH, Spartano NL, Stokes A, Tirschwell DL, Tsao CW, Turakhia MP, VanWagner LB, Wilkins JT, Wong SS, Virani SS, American Heart Association Council on Epidemiology and Prevention Statistics Committee and Stroke Statistics Subcommittee (2019) Heart disease and stroke statistics-2019 update: a report from the American Heart Association. Circulation 139:e56-e528. https://doi.org/10.1161/ CIR.0000000000000659

3. Biendarra-Tiegs SM, Secreto FJ, Nelson TJ (2020) Addressing variability and heterogeneity of induced pluripotent stem cellderived cardiomyocytes. Adv Exp Med Biol 1212:1-29. https:// doi.org/10.1007/5584_2019_350

4. Boheler KR, Czyz J, Tweedie D, Yang H-T, Anisimov SV, Wobus AM (2002) Differentiation of pluripotent embryonic stem cells into cardiomyocytes. Circ Res 91:189-201. https://doi.org/10. 1161/01.res.0000027865.61704.32

5. Boheler KR, Poon ENY (2021) Cell surface markers for immunophenotyping human pluripotent stem cell-derived cardiomyocytes. Pflügers Arch - Eur J Physiol. https://doi.org/10.1007/ s00424-021-02549-8

6. Burridge PW, Keller G, Gold JD, Wu JC (2012) Production of De Novo cardiomyocytes: human pluripotent stem cell differentiation and direct reprogramming. Cell Stem Cell 10:16-28. https://doi. org/10.1016/j.stem.2011.12.013

7. Doetschman TC, Eistetter H, Katz M, Schmidt W, Kemler R (1985) The in vitro development of blastocyst-derived embryonic stem cell lines: formation of visceral yolk sac, blood islands and myocardium. J Embryol Exp Morphol 87:27-45

8. Gepstein L (2002) Derivation and potential applications of human embryonic stem cells. Circ Res 91:866-876. https://doi.org/10. 1161/01.RES.0000041435.95082.84

9. Giannetti F, Benzoni P, Campostrini G, Milanesi R, Bucchi A, Baruscotti M, Dell'Era P, Rossini A, Barbuti A (2021) A detailed characterization of the hyperpolarization-activated "funny" current (If) in human-induced pluripotent stem cell (iPSC)-derived cardiomyocytes with pacemaker activity. Pflügers Arch - Eur J Physiol. https://doi.org/10.1007/s00424-021-02571-w

10. Hoes MF, Bomer N, van der Meer P (2019) Concise review: the current state of human in vitro cardiac disease modeling: a focus on gene editing and tissue engineering. Stem Cells Transl Med 8:66-74. https://doi.org/10.1002/sctm.18-0052

11. Kadota S, Pabon L, Reinecke H, Murry CE (2017) In vivo maturation of human induced pluripotent stem cell-derived cardiomyocytes in neonatal and adult rat hearts. Stem Cell Reports 8:278289. https://doi.org/10.1016/j.stemcr.2016.10.009

12. Kannan S, Kwon C (2019) Regulation of cardiomyocyte maturation during critical perinatal window. J Physiol 119:441-516. https://doi.org/10.1113/JP276754

13. Kelly MI, Albahrani M, Castro C, Poon E, Yan B, Littrell J, Waas M, Boheler KR, Gundry RL (2021) Importance of evaluating protein glycosylation in pluripotent stem cell-derived cardiomyocytes for research and clinical applications. Pflügers Arch - Eur J Physiol. https://doi.org/10.1007/s00424-021-02554-x

14. Kilpinen H, Goncalves A, Leha A, Afzal V, Alasoo K, Ashford S, Bala S, Bensaddek D, Casale FP, Culley OJ, Danecek P, Faulconbridge A, Harrison PW, Kathuria A, McCarthy D, McCarthy SA, Meleckyte R, Memari Y, Moens N, Soares F, Mann A, Streeter I, 
Agu CA, Alderton A, Nelson R, Harper S, Patel M, White A, Patel SR, Clarke L, Halai R, Kirton CM, Kolb-Kokocinski A, Beales P, Birney E, Danovi D, Lamond AI, Ouwehand WH, Vallier L, Watt FM, Durbin R, Stegle O, Gaffney DJ (2017) Common genetic variation drives molecular heterogeneity in human iPSCs. Nature 546:370-375. https://doi.org/10.1038/nature22403

15. King O, Sunyovszki I, Terracciano CM (2021) Vascularisation of pluripotent stem cell-derived myocardium: biomechanical insights for physiological relevance in cardiac tissue engineering. Pflügers Arch - Eur J Physiol. https://doi.org/10.1007/s00424-021-02557-8

16. Laflamme MA, Murry CE (2011) Heart regeneration. Nature 473:326-335. https://doi.org/10.1038/nature10147

17. Li Q, Wang J, Wu Q, Cao N, Yang H-T (2020) Perspective on human pluripotent stem cell-derived cardiomyocytes in heart disease modeling and repair. Stem Cells Transl Med 9:1121-1128. https://doi.org/10.1002/sctm.19-0340

18. Liu G, Liu Z, Cao N (2021) Human pluripotent stem cell-based cardiovascular disease modeling and drug discovery. Pflügers Arch - Eur J Physiol. https://doi.org/10.1007/s00424-021-02542-1

19. Liu Y-W, Chen B, Yang X, Fugate JA, Kalucki FA, FutakuchiTsuchida A, Couture L, Vogel KW, Astley CA, Baldessari A, Ogle J, Don CW, Steinberg ZL, Seslar SP, Tuck SA, Tsuchida H, Naumova AV, Dupras SK, Lyu MS, Lee J, Hailey DW, Reinecke H, Pabon L, Fryer BH, MacLellan WR, Thies RS, Murry CE (2018) Human embryonic stem cell-derived cardiomyocytes restore function in infarcted hearts of non-human primates. Nat Biotechnol 36:597-605. https://doi.org/10.1038/nbt.4162

20. Luo X-L, Zhang P, Liu X, Huang S, Rao S-L, Ding Q, Yang H-T (2021) Myosin light chain 2 marks differentiating ventricular cardiomyocytes derived from human embryonic stem cells. Pflügers Arch - Eur J Physiol. https://doi.org/10.1007/s00424-021-02578-3

21. Murphy SA, Chen EZ, Tung L, Boheler KR, Kwon C (2021) Maturing heart muscle cells: mechanisms and transcriptomic insights. Seminars in Cell and Developmental Biology 02 May Online ahead of print. https://doi.org/10.1016/j.semcdb.2021.04. 019

22. Murphy SA, Miyamoto M, Kervadec A, Kannan S, Tampakakis E, Kambhampati S, Lin BL, Paek S, Andersen P, Lee D-I, Zhu R, An SS, Kass DA, Uosaki H, Colas AR, Kwon C (2021) PGC1/PPAR drive cardiomyocyte maturation at single cell level via YAP1 and SF3B2. Nat Commun 12:1648. https://doi.org/10. 1038/s41467-021-21957-z

23. Murry CE, Keller G (2008) Differentiation of embryonic stem cells to clinically relevant populations: lessons from embryonic development. Cell 132:661-680. https://doi.org/10.1016/j.cell. 2008.02.008

24. Musunuru K, Sheikh F, Gupta RM, Houser SR, Maher KO, Milan DJ, Terzic A, Wu JC (2018) Induced pluripotent stem cells for cardiovascular disease modeling and precision medicine: a scientific statement from the American Heart Association. Circ Genom Precis Med 11:e000043. https://doi.org/10.1161/HCG.0000000000 000043

25. O'Brien TX, Lee KJ, Chien KR (1993) Positional specification of ventricular myosin light chain 2 expression in the primitive murine heart tube. Proc Natl Acad Sci U S A 90:5157-5161. https://doi.org/10.1073/pnas.90.11.5157

26. Pan Z, Ebert A, Liang P (2020) Human-induced pluripotent stem cells as models for rare cardiovascular diseases: from evidence-based medicine to precision medicine. PflügersArch Eur J Physiol. https://doi.org/10.1007/s00424-020-02486-y

27. Querdel E, Reinsch M, Castro L, Köse D, Bähr A, Reich S, Geertz B, Ulmer B, Schulze M, Lemoine MD, Krause T, Lemme M, Sani J, Shibamiya A, Stüdemann T, Köhne M, Bibra CV, Hornaschewitz N, Pecha S, Nejahsie Y, Mannhardt I, Christ T, Reichenspurner H, Hansen A, Klymiuk N, Krane M, Kupatt C, Eschenhagen T, Weinberger F (2021) Human engineered heart tissue patches remuscularize the injured Heart in a dose-dependent manner. Circulation 143:1991-2006. https://doi.org/10.1161/ CIRCULATIONAHA.120.047904

28. Robertson C, Tran DD, George SC (2013) Concise review: maturation phases of human pluripotent stem cell-derived cardiomyocytes. Stem Cells 31:829-837. https://doi.org/10.1002/stem.1331

29. Rubart M, Field LJ (2008) Stem cell differentiation: cardiac repair. Cells Tissues Organs 188:202-211. https://doi.org/10.1159/00011 2846

30. Svobodova B, Jelinkova S, Pesi M, Beckerova D, Lacampagne A, Meli AC, Rotreki V (2021) Cellular pathology of the human heart in Duchenne muscular dystrophy (DMD): lessons learned from in vitro modeling. Pflugers Arch - Eur J Physiol. https://doi.org/ 10.1007/s00424-021-02589-0

31. Takahashi K, Tanabe K, Ohnuki M, Narita M, Ichisaka T, Tomoda K, Yamanaka S (2007) Induction of pluripotent stem cells from adult human fibroblasts by defined factors. Cell 131:861-872. https://doi.org/10.1016/j.cell.2007.11.019

32. Thomson JA, Itskovitz-Eldor J, Shapiro SS, Waknitz MA, Swiergiel JJ, Marshall VS, Jones JM (1998) Embryonic stem cell lines derived from human blastocysts. Science 282:1145-1147. https:// doi.org/10.1126/science.282.5391.1145

33. Uosaki H, Cahan P, Lee DI, Wang S, Miyamoto M, Fernandez L, Kass DA, Kwon C (2015) Transcriptional landscape of cardiomyocyte maturation. Cell Rep 13:1705-1716. https://doi.org/10. 1016/j.celrep.2015.10.032

34. Vermersch E, Jouve C, Hulot J-S (2020) CRISPR/Cas9 gene-editing strategies in cardiovascular cells. Cardiovasc Res 116:894907. https://doi.org/10.1093/cvr/cvz250

35. Wang Y, Lei W, Yang J, Ni X, Ye L, Shen Z, Hu S (2021) The updated view on induced pluripotent stem cells for cardiovascular precision medicine. Pflugers Arch - Eur J Physiol. https://doi.org/ 10.1007/s00424-021-02530-5

36. Wobus AM, Boheler KR (2005) Embryonic stem cells: prospects for developmental biology and cell therapy. Physiol Rev 85:635678. https://doi.org/10.1152/physrev.00054.2003

37. Wobus AM, Wallukat G, Hescheler J (1991) Pluripotent mouse embryonic stem cells are able to differentiate into cardiomyocytes expressing chronotropic responses to adrenergic and cholinergic agents and $\mathrm{Ca} 2+$ channel blockers. Differentiation 48:173-182. https://doi.org/10.1111/j.1432-0436.1991.tb00255.x

Publisher's note Springer Nature remains neutral with regard to jurisdictional claims in published maps and institutional affiliations. 